\title{
BINDUNGSTRAUMA: KONZEPTE, URSACHEN UND MECHANISMEN IN INTIMEN BEZIEHUNGEN
}

\section{THEORETISCHE PRÜFUNG}

FONSECA, Nicole de Queiroz Lima1', OLIVEIRA, Bruno Quintino de ${ }^{2}$

FONSECA, Nicole de Queiroz Lima. OLIVEIRA, Bruno Quintino de. Bindungstrauma: Konzepte, Ursachen und Mechanismen in intimen Beziehungen. Revista Científica Multidisciplinar Núcleo do Conhecimento. Jahr. 06, Hrsg. 11, Vol. 06, S. 60-78. November 2021. ISSN: 2448-0959, Zugangslink: https://www.nucleodoconhecimento.com.br/psychologie/bindungstrauma, DOI: 10.32749/nucleodoconhecimento.com.br/psychologie/bindungstrauma
"Não te quero senão porque te quero
e de querer-te a não querer-te chego
e de esperar-te quando não te espero
passa meu coração do frio ao fogo.
Quero-te apenas porque a ti eu quero,
a ti odeio sem fim e, odiando-te, te suplico,
e a medida do meu amor viajante
é não ver-te e amar-te como um ego...".
(PABLO NERUDA - SONETO LXVI).

\footnotetext{
${ }^{1}$ Psychologiestudent. ORCID: 0000-0003-1739-9532

2 Doktorand in Psychoanalytischer Theorie - UFRJ, Master in Klinischer Psychologie - PUC RJ, Spezialist für psychische Gesundheit - HU/UFJF, Abschluss in Psychologie - UFF. ORCID: 0000-0002-7956-7899
}

RC: 101132

Verfügbar in:

https://www.nucleodoconhecimento.com.br/psychologie/bindungstrauma 


\section{ZUSAMMENFASSUNG}

Wenn nach einem gewalttätigen Vorfall starke emotionale Bindungen aufgebaut werden, um zwei Menschen zu verbinden, kommt es zur Installation eines Traumas, das in der Literatur als Bindungstrauma bezeichnet wird. Es ist eine emotionale Abhängigkeit zwischen zwei Menschen, in einer Beziehung, die durch Zeiten des Missbrauchs, der Gewalt und des Machtungleichgewichts gekennzeichnet ist, mit Bindungen intensiver Verbindung, kognitiver Verzerrung und Verhaltensstrategien beider Individuen, die paradoxerweise die Bindung stärken und aufrechterhalten, was sich widerspiegelt im Teufelskreis der Gewalt. Allerdings mangelt es in Brasilien an Literatur und wissenschaftlicher Forschung zu diesem Thema, was die Notwendigkeit einer genauen und eingehenden Untersuchung unterstreicht, da die Ursachen und Auswirkungen von Bindungstrauma nicht genau klar sind, was das Verständnis und die Behandlung von Individuen erschwert sowie ihre Genesung, ein langsamer und schmerzhafter Prozess. Daher orientiert sich diese Forschung an der Frage: Was ist Bindungstrauma und was sind seine Mechanismen? Daher wurde es als allgemeines Ziel definiert, das Bindungstrauma in romantischen Beziehungen zu analysieren, um die neuropsychobiologischen Prozesse von Traumata, die in intimen Beziehungen entstehen, zu beleuchten und die Gründe zu untersuchen, warum die Opfer in pathologischen Beziehungen verbleiben -traumatische Belastungsstörung bei Missbrauchsopfern in romantischen Beziehungen als Generatoren von Bindungstrauma. Zur Kontextualisierung, Hinterfragung und Validierung des theoretischen Rahmens, der in der durchgeführten Forschung verwendet wurde, wurde eine Literaturrecherche oder bibliografische Überprüfung verwendet, die auf der Lektüre, Auswahl und Analyse von Texten unterschiedlicher Genres, wie Aufzeichnungen, Rezensionen und wissenschaftlichen Artikeln, basiert zu diesem Thema und die in Zeitschriften und Zeitungen in der Umgebung veröffentlicht wurden. Es wurde der Schluss gezogen, dass sich mehrere emotionale und neuropsychobiologische Faktoren als Ursache für das Bindungstrauma in einer Beziehung darstellen.

RC: 101132

Verfügbar in:

https://www.nucleodoconhecimento.com.br/psychologie/bindungstrauma 
Schlüsselwörter: Trauma, Bindungstrauma, Posttraumatische Belastungsstörung, Stockholm-Syndrom, Gewalt in intimen Beziehungen.

\section{EINLEITUNG}

Diese Forschung zielt darauf ab, Bindungstrauma in intimen Beziehungen zu analysieren, in denen es ein bestimmtes Verhaltensmuster gibt, das auf Missbrauch durch einen der Partner basiert. Es wird viel über psychologischen und sexuellen Missbrauch in affektiven Beziehungen und die Auswirkungen, die sie verursachen, gesagt, jedoch sind die Hauptziele dieser Forschung von zwei unterschiedlichen und miteinander verbundenen Naturen: a) den neuropsychobiologischen Prozessen von Traumata, die in intimen Beziehungen erzeugt werden, Licht zu geben; und b) die Gründe untersuchen, warum Opfer in pathologischen Beziehungen bleiben.

Der Mangel an Literatur und wissenschaftlicher Forschung in diesem Bereich in Brasilien bringt die Notwendigkeit einer verfeinerten und eingehenden Studie ans Licht, da die Ursachen und Auswirkungen von Bindungstrauma immer noch nicht genau klar sind, was das Verständnis und die Behandlung von Individuen sowie ihre Genesung zu einem langsamen und schmerzhaften Prozess macht. Traumaopfer müssen lernen, Beziehungen aufzubauen, die nicht auf Angst und Machtmissbrauch basieren, auch wenn ein solcher Machtmissbrauch als natürlich und sicher wahrgenommen wird (BLOOM, 1999).

Im gesunden Menschenverstand gibt es die weit verbreitete Vorstellung, dass Menschen, die in Beziehungen bleiben, die als missbräuchlich angesehen werden, dies ausschließlich durch Probleme der finanziellen, sozialen Abhängigkeit, des geringen Selbstwertgefühls und / oder der psychologischen Abhängigkeit motiviert tun. Obwohl solche Aspekte relevant sein können, deuten Studien darauf hin, dass Bindungstrauma tatsächlich das sind, was Probanden in prekären Situationen in ihren liebevollen Beziehungen hält. In seiner Arbeit "The Betrayal Bond" erklärt Carnes (2019):

RC: 101132

Verfügbar in:

https://www.nucleodoconhecimento.com.br/psychologie/bindungstrauma 
Trauma bonds são formas disfuncionais de ligação que ocorrem na presença de perigo, vergonha e abuso. Esses relacionamentos são bem diferentes das ligações que ocorrem naturalmente quando experimentamos um evento traumático com outras pessoas e o resultado geralmente é uma união ou estreitamento de uma ligação como método de sobrevivência. Trauma bonds, por outro lado, acontecem quando nos apegamos à exata pessoa que é a fonte do medo, vergonha e abuso. Este tipo de ligação não facilita a recuperação e resiliência, sabotando tais qualidades em nós, e frequentemente deixam um impacto a longo prazo, geralmente envolvendo sedução e traição[3]. (CARNES, 2019, p. 121).

Traumatic (oder traumatische) Bindungen entstehen, wenn starke emotionale Bindungen aufgebaut werden, um zwei Menschen zu verbinden, nach einem Vorfall, bei dem eine Person angreift, gewalttätiges Verhalten ausdrückt, missbräuchlich, einschüchternd und bedrohlich ist. Es ist eine emotionale Abhängigkeit zwischen zwei Menschen, in einer Beziehung, die durch Perioden von Missbrauch, Gewalt und Machtungleichgewicht gekennzeichnet ist. Die Wurzel dieser Bindung ist gekennzeichnet durch Gefühle intensiver Verbindung, kognitiver Verzerrung und Verhaltensstrategien beider Individuen, die paradoxerweise die Bindung stärken und aufrechterhalten, was sich im Teufelskreis der Gewalt (DEYOUNG; LOWRY, 1992).

Aus physiologischer Sicht kann man sagen, dass das menschliche Gehirn in seiner ganzen Komplexität während des psychosozialen Prozesses der Entstehung des Bindungstrauma aktiviert wird. Autoren wie Van Der Kolk (1989, 1991, 1994, 2001), Simonič und Osewska (2019), Fisher (2010) und Bloom (1999) postulieren Studien, die belegen, dass Menschen, die häufig oder länger missbraucht werden, sowie Kindheitstraumata häufiger in Beziehungen mit Bindungstrauma verwickelt sind , wegen eines Belohnungsmechanismus im Organismus, der nach Beendigung der Krisensituation die Wiederholung bestimmter durch die Ausschüttung von Endorphinen erzeugter Empfindungen anstrebt.

Sekunde. Bloom (1999), in seiner Arbeit "Trauma Theory Abbreviated", funktioniert ein solcher Mechanismus ähnlich wie in Organismen von Menschen, die auf Betäubungsmittel angewiesen sind:

RC: 101132

Verfügbar in:

https://www.nucleodoconhecimento.com.br/psychologie/bindungstrauma 
[...]essas substâncias mágicas chamadas endorfinas são parte do funcionamento normal e corriqueiro, mas elas são especialmente importantes em momentos de estresse. Novamente, se olharmos para a evolução, faz sentido. Endorfinas não apenas acalmam a ansiedade, melhoram nosso humor e diminuem a agressividade, mas também são ótimos analgésicos já que são relacionados com a morfina e a heroína. Portanto, em tempos de estresse, elas propiciam alívio para a dor o suficiente para que não sejamos incapacitados por ferimentos que poderiam nos impedir de escapar do perigo. Se as pessoas são expostas a raros episódios de intenso estresse, elas possuem menos chances de mostrarem quaisquer mudanças em seu sistema bioquímico. O problema reside nas pessoas que são expostas repetidamente a experiências de estresse prolongado. Tais pessoas, geralmente crianças, são expostas a altos níveis de endorfinas frequentemente. Uma hipótese é a de que os indivíduos podem se tornar 'viciados' em suas próprias endorfinas e, como resultado, só se sentem calmas quando estão sob stress, com medo, irritáveis e hiper excitadas quando o estresse é aliviado, bastante similar a pessoas em abstinência de heroína. Isso é chamado de 'vício em trauma'[4]. (BLOOM, 1999, p. 9).

Mehrere im menschlichen Körper gebildete Hormone sind an der sogenannten "Trauma Sucht" beteiligt, die eine Bindung zum Täter stärkt oder induziert, wie zum Beispiel Oxytocin - das Bindungen und Zuneigung verursacht; endogene Opioide, die für Lust, Schmerz, Abstinenz und Abhängigkeit verantwortlich sind; CorticotropinReleasing-Faktor, der Entzug und Stress verursacht; das Dopamin - erzeugt das Verlangen, das Verlangen, die Suche. Bei so vielen neurochemischen Faktoren in fehlregulierten Zuständen ist es für das Subjekt extrem schwierig, seine Emotionen zu modulieren und Entscheidungen zu treffen (BURKETT; YOUNG, 2012).

Andere Hormone beeinflussen die Reaktion (oder das Fehlen derselben) in der Person angesichts einer Situation der Gewalt und / oder Beständigkeit von Individuen in einer missbräuchlichen Beziehung. Die Wahrnehmung, dass bestrafung unmittelbar bevorsteht, aktiviert zwei Gehirnmechanismen im Subjekt: einen, der das vorherige Verhalten hemmt, was angeblich den Grund für die Bestrafung erzeugt hat; und die andere, die die Aufregung angesichts der erzeugten gewalttätigen Situation erhöht. Das zentrale Nervensystem aktiviert unter diesen Umständen die Auslöser für

RC: 101132

Verfügbar in:

https://www.nucleodoconhecimento.com.br/psychologie/bindungstrauma 
Notfälle und erzeugt die Verhaltens- und kognitiven Reaktionen des Probanden, um sich vor einem möglichen Angriff zu schützen (BURKETT; YOUNG, 2012).

Mehrere Studien zeigen, dass serotoninerge Antagonisten die Aggressivität als Reaktion auf Stress und Hyperreaktivität als Reaktion auf Reize erhöhen, aber die Unterdrückung von Verhaltensweisen, die durch Bestrafung verursacht werden, wird durch Serotoninblocker umgekehrt (VAN DER KOLK; SAPORTA, 1991), die die Hypothese aufwirft, dass der niedrige serotonerge Spiegel bei Opfern von Traumata und posttraumatischem Stress für die Fortsetzung von Notfallreaktionen auf kleinere Reize verantwortlich ist, auch wenn die Ursache des Traumas gestoppt wird.

Van Der Kolk und Saporta (1991) erklären auch, dass die Schwere posttraumatischer Stressfälle mit dem Cortisolspiegel im Körper des Probanden zusammenhängt, was die Hypothese stützt, dass es einen chronischen Anstieg des Cortisols bei Personen gibt, die von posttraumatischem Stress betroffen sind.

Der Begriff "Stockholm syndrom" wurde 1973 nach dem Ereignis eines Banküberfalls in Stockholm, Schweden, geprägt, bei dem Kriminelle und Geiseln sechs Tage lang eingesperrt blieben (MARTIN, 2005). Es gibt Berichte, dass die Opfer in diesen sechs Tagen Empathie, Zuneigung und Komplizenschaft mit ihren Entführern entwickelt haben. Weil verstanden wurde, dass es sich um eine Verbindung zwischen Täter und Opfer handelte, erschien eine solche Beziehung den aufmerksamsten Augen seltsam, und der Psychiater und Kriminologe Nils Bejerot taufte dieses Phänomen des Stockholm-Syndroms.

Bei der Untersuchung anderer Erfahrungen in der Geschichte der psychischen Gesundheit im Westen wurde festgestellt, dass dieses Phänomen besonders häufig in einigen religiösen Kulten, in Beziehungen zu möglichen Kriegsgefangenen, in familiären Beziehungen mit Fällen von Kindern, die Opfer von Missbrauch oder Inzest wurden, unter anderen Gruppen vorsah. Es wurde auch festgestellt, dass

RC: 101132

Verfügbar in:

https://www.nucleodoconhecimento.com.br/psychologie/bindungstrauma 
dieses Syndrom auch in liebevollen Beziehungen entwickelt wird, in denen das Opfer eine traumatische Verbindung zu seinem Täter herstellt (GEORGE, 2015).

Nach Graham et al. (1995) gibt es vier Vorläufer des Stockholm syndrom: a) das lebensbedrohliche Gefühl; b) scheinbare Freundlichkeit; c) Isolation; und d) die offensichtliche Unfähigkeit, der Situation zu entkommen.

Das Stockholm-Syndrom ist daher ein Abwehrmechanismus für das Individuum, um mit solchen Faktoren umzugehen, zu denen kognitive Verzerrungen und die Wahrnehmung der Realität gehören können (GEORGE, 2015).

Solche Verzerrungen werden auch als kognitive Dissonanzen bezeichnet, von Leon Festinger in seinem Werk " $A$ Theory Of Cognitive Dissonance" (1957). Für den Autor existieren diese Dissonanzen, wenn es einen Konflikt oder eine Inkongruenz zwischen verschiedenen Überzeugungen gibt oder wenn das Subjekt mit Informationen konfrontiert wird, die seine früheren Überzeugungen widerlegen. Kognitive Dissonanz erzeugt einen unangenehmen psychologischen Zustand, der die Schaffung von Gehirnmechanismen in Form von Strategien zur Verringerung von Beschwerden motiviert.

Wendet man eine solche Theorie auf eine missbräuchliche Beziehung an, erstellt das Opfer am Ende bestimmte Ausarbeitungen oder sogar Rechtfertigungen für das Verhalten seines Partners, da seine Hauptüberzeugung darin besteht, dass er nicht missbraucht wird. Selbst angesichts von Fakten und Beweisen ist das Opfer nicht in der Lage, seine kognitive Dissonanz zu widerlegen. Angesichts des obigen Bildes können wir erkennen, dass das Verbleiben in einer missbräuchlichen Beziehung kein rein psychologisches oder soziales Problem ist. Die Identifizierung des Auftretens von Traumata in der Kindheit und während des gesamten Lebens ist von grundlegender Bedeutung, um zu verstehen, wie neurologische Muster und traumatische Bindungen aus Traumata gebildet werden und während des gesamten Lebens bestehen bleiben (GEORGE, 2015).

RC: 101132

Verfügbar in:

https://www.nucleodoconhecimento.com.br/psychologie/bindungstrauma 
Personen, die seit ihrer Kindheit aufgrund von familiärem Missbrauch, Verlassenheit, Inzest und anderen gewalttätigen Situationen einem hohen Stress- und Cortisolspiegel ausgesetzt sind, suchen unbewusst Menschen, die zu Verhaltensweisen neigen, die als missbräuchlich gelten und die zu Konflikten und dem Gefühl führen können von Angst, Scham und Missbrauch, an die sie gewöhnt sind und dass ihr Gehirn den Hormonspiegel modulieren muss (BLOOM, 1999).

Die psychologischen und organischen Reaktionen, denen Personen ausgesetzt sind, die sich irgendwann im Leben dafür entscheiden, eine als missbräuchlich angesehene Beziehung zu verlassen, ähneln denen, die Suchtkranke beim Entzug erleben, was starke Beweise für das Vorhandensein eines Mechanismus liefert, der die menschliche Hormonproduktion in Beziehung setzt mit Bindungstrauma (BLOOM, 1999).

Aus der gegenwärtigen Experformance wird die Relevanz dieser Studie verteidigt, die darauf abzielt, solche Dynamiken zu untersuchen, damit sich Individuen, die Geiseln dieser Verbindungen sind, tatsächlich effektiv von Traumata - vergangenheit und gegenwart - erholen und so verhindern können, dass sich der Zyklus in der Zukunft wiederholt.

Die Notwendigkeit, das Verständnis des Konzepts des Bindungstrauma für die Aufnahme und Behandlung von Individuen zu vertiefen sowie seine biopsychosoziale Funktionsweise und kurz- und langfristigen Auswirkungen zu verstehen, machen die Erforschung dieses Themas von klinischer, theoretischer, sozialer und politischer Bedeutung.

Gewalt, körperlich und emotional, in intimen Beziehungen, ist ein umfassendes und schädliches Phänomen, das in der gesamten Gesellschaft ohne Geschlechtsdifferenzierung auftritt und Männer, Frauen, intersexuelle Menschen und andere Geschlechtsidentitäten betrifft und zu verschiedenen psychologischen, sozialen und sogar körperlichen Problemen führt.

RC: 101132

Verfügbar in:

https://www.nucleodoconhecimento.com.br/psychologie/bindungstrauma 
Das Verständnis der neuropsychologischen Aspekte bei Bindungstrauma und "Traumasucht" ist eine Aufgabe von größter Bedeutung, da es aus solchen Kompetenzen möglich sein wird, klinische Interventionsstrategien in solchen Fällen zu entwickeln. Als Ausgangspunkt für die zukünftige Konstruktion eines klinischen Protokolls wird verstanden, dass physiologische Stabilität nach Bloom (1999) nicht erreicht werden kann, während sich das Individuum auf einer ständigen Achterbahn von Reizen und Reaktionen befindet.

Aus diesen Gründen versucht diese Forschung, eine neue Perspektive auf missbräuchliche Beziehungen und die Dauerhaftigkeit von Probanden in diesen Situationen zu schaffen, da der Mangel an Studien und wissenschaftlichen Publikationen in Brasilien Ansätze zur Situation veraltet oder veraltet machen kann.

Diese Forschung wird von der folgenden Leitfrage geleitet: Was ist bindungstrauma und was sind seine Mechanismen? Das allgemeine Ziel dieser Arbeit ist es daher, bindungstrauma in liebevollen Beziehungen $\mathrm{zu}$ analysieren, um die neuropsychobiologischen Traumaprozesse, die in intimen Beziehungen erzeugt werden, zu gebären und die Gründe zu untersuchen, warum Opfer in pathologischen Beziehungen bleiben.Konkret soll es: a) Erklären, was ein bindungstrauma ist und wie es in einer missbräuchlichen Beziehung auftritt; b) Den Grund dafür zu analysieren, dass ein bestimmtes Profil von Missbrauchsopfern in diesen Beziehungen verbleibt; und c) Erklären Sie die Funktionsweise des StockholmSyndroms und posttraumatischen Stresses bei Missbrauchsopfern in liebevollen Beziehungen als Faktoren, die ein bindungstrauma erzeugen.

Die in dieser Studie verwendete Methodik ist Literaturübersicht oder Literaturübersicht. Diese Art von Methodik hat als Zweck die Konstruktion einer Kontextualisierung für das Problem sowie die Analyse der Möglichkeiten, die in der Literatur für die Konzeption des theoretischen Rahmens der Forschung vorhanden sind.

RC: 101132

Verfügbar in:

https://www.nucleodoconhecimento.com.br/psychologie/bindungstrauma 
Dazu wurden Lesungen von Texten verschiedener Genres gemacht, wie Aufzeichnungen, Rezensionen und wissenschaftliche Artikel zum Thema, die in Zeitschriften und Zeitungen in der Region veröffentlicht wurden. Dann wurde das durch die bibliographische Umfrage gesammelte Material organisiert und analysiert. Diese Analyse trug zur Kontextualisierung, Problematisierung und Validierung des theoretischen Rahmens bei, der in der durchgeführten Forschung verwendet wurde.

\section{GEWALT UND INTIME BEZIEHUNGEN}

Was treibt jemanden dazu, die Person, von der er sagt, dass sie sie liebt, Gewalt aus zu unterwerfen?Die Gründe können variieren, z. B. Drogenmissbrauch; frühere neurologische Erkrankungen, die das Verhalten beeinflussen können; Persönlichkeitsstörungen, wie antisoziale Persönlichkeitsstörung; BorderlinePersönlichkeitsstörung; und narzisstische Persönlichkeitsstörung.

So unterschiedlich die Gründe auch sein mögen, das Ergebnis ist in der Regel dasselbe: Das Opfer leidet unter den Folgen des Missbrauchs. Manchmal werden diese Partner nicht in der Lage sein, aus solchen Beziehungen herauszukommen. Die Familie, Freunde und die Menschen um diese Opfer herum können ihnen sagen, dass sie die Beziehung beenden und aus der Situation des Leidens herauskommen sollen, indem sie an die Logik appellieren; Die natürliche Funktion des Gehirns kann dies jedoch verhindern.

Menschen, die Opfer eines Traumas sind, setzen sich scheinbar zwanghaft Situationen aus, die sich auf das ursprüngliche Trauma beziehen. Diese Verhaltensweisen, die traumatisierten, werden selten bewusst als etwas verstanden, das mit Erfahrungen zusammenhängt, die früh in ihrem Leben aufgetreten sind. Laut Van Der Kolk (1989) sind Menschen Wesen, die auf soziale Unterstützung angewiesen sind, um ein Gefühl von persönlicher Bedeutung, Sicherheit, Macht und Kontrolle zu schaffen. Sogar unsere biologische Reifung wird davon beeinflusst, wie bindungen seit der Kindheit gebildet wurden. Ein Trauma tritt auf, wenn das Subjekt

RC: 101132

Verfügbar in:

https://www.nucleodoconhecimento.com.br/psychologie/bindungstrauma 
sowohl intern als auch extern nicht über die geeigneten Werkzeuge verfügt, um mit externen Bedrohungen umzugehen.

Körperliche und emotionale Reife sowie angeborene physiologische Variationen im Prozess der Reaktion und Wahrnehmung von Gefahren sind grundlegend für die Fähigkeit des Individuums, mit äußeren Bedrohungen umzugehen. Die Anwesenheit von Betreuern, die Sicherheit bieten, ist von größter Bedeutung für die Modulation der physiologischen Erregung bei Kindern, sowie ihre Abwesenheit kann dazu führen, dass sie In dieser Erregung Auf- und Aben erleben, die physiologisch aversiv und desorganisierend sind. Die Figur der Bezugsperson, auf die sich das Kind verlassen kann, um mit Situationen umzugehen, auf die es nicht vorbereitet ist, ist für inn von grundlegender Bedeutung, um biologische Ressourcen zu entwickeln, um lebenslangen Bedrohungen zu begegnen (VAN DER KOLK, 1989).

Nach Van Der Kolk (1989), wenn Menschen, die die Quelle des körperlichen und emotionalen Schutzes und der Ernährung sein sollten, zur Quelle der Gefahr werden, vor der das Kind geschützt werden sollte, neigen sie dazu, sich neu anzupassen und eine innere Bedeutung von Sicherheit zu etablieren. Anstatt sich gegen ihre Betreuer zu wenden und die Hoffnung auf irgendeine Art von Schutz zu verlieren, geben sie sich selbst die Schuld und werden ängstlich, extrem angebunden, ängstlich und gehorsam.

Die Verletzlichkeit der Person, die zu Beginn des Lebens opfer eines Traumas wurde, macht sie anfällig für Traumata, wiederum im Erwachsenenalter, da sie aufgrund ihrer seit ihrer Kindheit veränderten kognitiven Struktur anfällig für ein solches Ereignis wird. Für Van der Kolk (1989, S. 391) "beginnen Menschen, die in der Kindheit Gewalt und Vernachlässigung ausgesetzt sind, als Teil des Lebens auf sie zu warten[5]". In diesem Zusammenhang wird das Bindungstrauma anfällig für die Eingewöhnung.

RC: 101132

Verfügbar in:

https://www.nucleodoconhecimento.com.br/psychologie/bindungstrauma 
Die neurobiologischen Veränderungen, die bei Missbrauchsopfern auftreten, scheinen denen der Trennungsphase einer nicht missbräuchlichen Beziehung ähnlich zu sein (FISHER et al., 2010). Opfer von Gewalt in intimen Beziehungen unterscheiden sich von anderen Opfern von Gewalt, weil sie dem Aggressor nahe stehen. Daher wird diese Art von Gewalt von einer besonderen emotionalen Dynamik begleitet, die auf der Bindung zwischen Aggressoropfern (SIMONIČ; OSEWSKA, 2019).

Wenn sich einer von uns verliebt und sich mit jemand Neuem verbindet, reagiert die Neurochemie des Belohnungssystems, um diese Bindung herzustellen. Unter Umständen des Missbrauchs hat das Gehirn die gleiche Bindung, die jeder für jemanden haben würde, den er liebt. Für Missbrauchsopfer ist der geliebte Menschen jedoch nicht sicher und die Beziehung ist nicht stabil (BURKETT; YOUNG, 2012).

Was neurobiologisch in einer Beziehung passiert, die aus einem Bindungstrauma besteht, unterscheidet sich nicht wesentlich von dem, was in einer gesunden Beziehung passiert. Der Hauptunterschied besteht darin, dass das menschliche Gehirn angesichts der Tatsache, dass es extrem empfindlich auf das reagiert, was in der Umgebung passiert, Chemikalien als Reaktion auf das missbräuchliche Verhalten des Partners freisetzt. Wenn er sich entfernt oder sich aggressiv verhält, wird es im Gehirn der Person mit einer Geschichte von Trauma und die eine missbräuchliche Beziehung lebt, eine differenzierte Reaktion geben, die jemand ohne Vorgeschichte von Trauma und in einer "normalen Beziehung" nicht erleben würde. Dies gilt auch für die Neurochemie des Gehirns mit endogenen Opioiden, Dopamin und Corticotrophin-Freisetzungsfaktor. (BURKETT; YOUNG, 2012).

\subsection{POSTTRAUMATISCHER STRESS}

Van der Kolk (1994) erklärt in seiner Arbeit "The Body Keeps Score: Memory and the evolving psychobiology of post-traumatic stress", die:

RC: 101132

Verfügbar in:

https://www.nucleodoconhecimento.com.br/psychologie/bindungstrauma 
O Transtorno de Estresse Pós-Traumático se desenvolve após a exposição a eventos muito angustiantes. O estresse intenso é acompanhado pela liberação de neuro-hormônios endógenos que respondem ao estresse, como cortisol, epinefrina e norepinefrina, vasopressina, ocitocina e opioides endógenos. Esses hormônios ajudam o organismo a mobilizar a energia necessária para lidar com o estresse, desde o aumento da liberação de glicose até o aprimoramento da função imunológica. Em um organismo que funciona bem, o estresse produz respostas hormonais rápidas e pronunciadas. No entanto, o estresse crônico e persistente inibe a eficácia da resposta ao estresse e induz dessensibilização[6]. (VAN DER KOLK, 1994, p. 4).

Posttraumatischer Stress kann durch fünf Kategorien von Symptomen identifiziert werden, sie sind: belastende, wiederkehrende und unwillkürliche aufdringliche Erinnerungen an das traumatische Ereignis; wiederkehrende belastende Träume, in denen der Inhalt und/ oder das Gefühl von Träumen mit dem traumatischen Ereignis zusammenhängen; dissoziative Reaktionen (z. B. flashbacks), bei denen das Individuum das Gefühl hat oder so tut, als ob das traumatische Ereignis erneut auftritt; intensive oder anhaltende psychische Belastung angesichts der Exposition gegenüber inneren oder äußeren Anzeichen, die einen Aspekt des traumatischen Ereignisses symbolisieren oder innen ähnlich sind; intensive physiologische Reaktionen auf innere oder äußere Anzeichen, die einen Aspekt des traumatischen Ereignisses symbolisieren oder innen ähnlich sind (AMERICAN PSYCHIATRIC ASSOCIATION, 2014).

Solche Symptome verursachen ein hohes Maß an Verwirrung und Leid für Überlebende, die nicht verstehen, wie sie plötzlich in ihrem eigenen Geist und Körper so außer Kontrolle geraten sind. Unerwartete Wut oder Tränen, Kurzatmigkeit, erhöhte Herzfrequenz, Zittern, Gedächtnisverlust, Konzentrationsprobleme, Schlaflosigkeit, Albträume und emotionale Taubheit können eine Identität und ein Leben entführen.

Laut Van der Kolk (2001) erfährt das menschliche Gehirn nach dem Trauma biologische Veränderungen, die es nicht erfahren hätte, wenn kein Trauma

RC: 101132

Verfügbar in:

https://www.nucleodoconhecimento.com.br/psychologie/bindungstrauma 
aufgetreten wäre. Die Auswirkungen dieser Veränderungen werden insbesondere durch drei Hauptderegulierungen der Gehirnfunktion verstärkt:

a) Überstimulierte Mandel: Nach einem Trauma besteht die Tendenz des Individuums, Emotionen als physischen Zustand und nicht als verbal kodierte Erfahrungen zu erleben.

b) Verminderter Hippocampus: Ein Anstieg des Stresshormons Cortisol, das für den Hippocampus toxisch ist, führt zu einer Verringerung der Größe, was auf einen Verlust der Zellmasse hindeutet. Dies macht es weniger effektiv bei der Herstellung der snatischen Verbindungen, die für die Speicherkonsolidierung erforderlich sind. Diese Unterbrechung hält Körper und Geist im reaktiven Modus stimuliert, da keines der Elemente die Nachricht erhält, dass sich die Bedrohung in der Vergangenheit geändert hat.

c) Lateralisierung: Van der Kolk (2001) berichtet in seiner Studie, dass es eine Tendenz zur Lateralisierung zur rechten Hemisphäre des Gehirns gibt, die für die Bewertung der emotionalen Bedeutung der erhaltenen Informationen und für die autonome und hormonelle Regulation der Reaktionen auf diese Reize verantwortlich ist. Das heißt, die rechte Hemisphäre des Gehirns ist hyperaktiviert, und im Gegensatz dazu hat der Bohrbereich - Teil der linken Hemisphäre, der für die Übersetzung persönlicher Erfahrungen in verbale Kommunikation verantwortlich ist eine Abnahme des Sauerstoffverbrauchs aufgrund der Exposition gegenüber Erinnerungen an das traumatische Ereignis.

Angesichts des oben Gesagten wird darauf hingewiesen, dass die Erinnerung an das traumatische Ereignis für das Gehirn so ist, als ob das Individuum die Situation wieder lebt und die mit dem Trauma verbundenen sensorischen Elemente sehen, hören und fühlen kann. Es gibt auch physiologische Beeinträchtigungen, wenn versucht wird, die traumatische Erfahrung durch verbale Kommunikation sowie Dissoziation von Gefühlen aufzudecken (VAN DER KOLK, 2001).

RC: 101132

Verfügbar in:

https://www.nucleodoconhecimento.com.br/psychologie/bindungstrauma 
Basierend auf der Psychobiologie des Traumas wird festgestellt, dass die Gehirnfunktion des Individuums inn daran hindert, eine missbräuchliche Beziehung als solche wahrnehmbar zu machen, da sein Gehirn und sein Körper an Stress und Vernachlässigung sowie Angst und Angst gewöhnt sind, die auch durch solche Beziehungen verursacht werden (VAN DER KOLK, 1989).

\subsection{STOCKHOLM-SYNDROM}

Graham et al. (1995) entwickelte die Stockholm-Syndrom-Theorie, die speziell mit Gewalt in einer intimen Beziehung verbunden ist, basierend auf der Psychologie und dem Verhalten von Gruppen wie Sektenmitgliedern, KZ-Häftlingen, Zivilisten in chinesischen kommunistischen Gefängnissen, Kriegsgefangenen, missbrauchten Kindern, Inzestopfer und Prostituierte, die Zuhälter besaßen. Sie argumentiert, dass vier Vorläufer für die Entwicklung des Stockholm-Syndroms notwendig sind: Wahrnehmung der Überlebensbedrohung, Wahrnehmung des Guten, Isolation und Wahrnehmung der Unfähigkeit zu fliehen.

Das Stockholm-Syndrom stellt einen Abwehrmechanismus für den Umgang mit diesen Faktoren dar, einschließlich kognitiver und wahrnehmungsbezogener Verzerrungen. Sechsundsechzig Punkte wurden von Graham (Verhaltensweisen, Einstellungen und Überzeugungen) als mit dem Syndrom verbunden aufgeführt. Basierend auf diesen Elementen entwickelte Graham eine 49-Punkte-Skala zur Messung des Stockholm-Syndroms in Beziehungen (GEORGE, 2015).

Auf der ursprünglichen Skala haben Graham et al. (1995) fand heraus, dass die Artikel in drei Kategorien weit verbreitet waren: zentrales Stockholm-Syndrom, psychische Schäden und Liebessucht.

Das zentrale Stockholm-Syndrom enthielt zentrale Aspekte der Stockholm-SyndromTheorie und beschrieb kognitive Verzerrungen und zwischenmenschliche Traumata. Dazu gehörten die Rationalisierung oder Minimierung des gewalttätigen Verhaltens

RC: 101132

Verfügbar in:

https://www.nucleodoconhecimento.com.br/psychologie/bindungstrauma 
eines Partners, das Selbstanklageverhalten angesichts von Partnerversagen oder Verhaltensweisen und die Identifizierung von Liebe im Kontext von Angst.

Psychische Schäden erfassten Depressionen, geringes Selbstwertgefühl und zwischenmenschliche Schwierigkeiten.

Liebesabhängigkeit war gekennzeichnet durch den starken Glauben, dass das Überleben selbst von der Zuneigung eines Partners, extremem Götzendienst und dem Glauben abhängt, dass es ohne einen Partner nichts zum Leben geben würde (GEORGE, 2015).

Romantische Liebe kann als Sucht betrachtet werden, da sie Merkmale der Sucht zeigt, wie zum Beispiel: die intensiv fokussierte Aufmerksamkeit des Subjekts in einem bevorzugten Objekt (in diesem Fall individuell), plötzliche und dekontextualisierte Stimmungsschwankungen, intensives und unkontrollierbares Verlangen, Besessenheit mit einem Objekt / Individuum, Zwang, Verzerrung der Realität, emotionale Abhängigkeit, Persönlichkeitsveränderungen, hohes Risiko zur Erfüllung von Wünschen, die mit diesem Objekt / Individuum verbunden sind, und der Verlust der Selbstbeherrschung angesichts des eigenen Verlangens. Romantische Liebe neigt dazu, eine konstruktive Form der Sucht zu sein, wenn sie erwidert wird, aber sie kann zu einer destruktiven Ergänzung werden, wenn sie abgelehnt wird (FISHER et al., 2010).

Mit dieser Dynamik im Kreislauf von Gewalt, Liebe und Ablehnung entsteht eine spezifische Form der Verbindung zwischen den beiden Partnern, die hauptsächlich auf emotionaler Dynamik und Vernetzung beruht, durch die das Opfer trotz seiner rationalen Anerkennung von Gewalt innig spürt, dass es nicht in der Lage ist, den missbräuchlichen Partner einfach zu verlassen, der wiederum ändert $\mathrm{lhr}$ Verhaltensmuster nicht. Anstatt die Beziehung zu beenden (und selbst wenn er es tut, kehrt er nach einer Weile zurück), stärkt das Opfer seine Bindung zum Aggressor. Auf diese Weise vertieft sich die Komplexität der missbräuchlichen

RC: 101132

Verfügbar in:

https://www.nucleodoconhecimento.com.br/psychologie/bindungstrauma 
Beziehung und der Kreislauf der Gewalt wiederholt sich (SIMONIČ; OSEWSKA, 2019).

Dutton und Painter $(1981,1993)$ beschrieben diese Art von Bindung als traumatic bonding bieten eine breitere Beschreibung der Reaktionsdynamik von Opfer und Täter mit vorhersehbaren und unvorhersehbaren Gewaltmustern und Reaktionen darauf.

\subsection{BINDUNGSTRAUMA}

Bindungstrauma ist die Bindung an eine missbräuchliche Beziehung und ihr Ergebnis ist eine traumatische affektive Bindung, die im Kreislauf der Gewalt entsteht, die auftritt, ohne dass das Individuum es nesselt. Opfer können die subtilen Anzeichen von Missbrauch ignorieren, wenn die Beziehung noch früh ist und sich des bevorstehenden emotionalen Missbrauchs nicht bewusst sind; Wenn sich die Beziehung jedoch ausdehnt und die Bindungen vertiefen, wird das Muster des gewalttätigen und missbräuchlichen Verhaltens eines Teils der Beziehung tendenziell klarer (DUTTON; PAINTER, 1993).

Im Allgemeinen sind zu Beginn missbräuchlicher Beziehungen Die Episoden des Missbrauchs mild und das Muster der Gewalt ist noch unklar. Darüber hinaus sagt der Angreifer, dass er es bereut und sich entschuldigt, und das Opfer akzeptiert sie, was die emotionale Bindung verstärkt. (DUTTON; PAINTER, 1993). Diese Phase kann nur ein frühes kurzes Stadium der missbräuchlichen Beziehung sein oder lange Dauern dauern, bis man in eine neue Phase des Gewaltzyklus eintritt.

Setzt man die Beziehung missbräuchlicher Natur fort, folgt die Entwicklung des Gewaltzyklus und die neue Phase ist durch die Zunahme des Missbrauchs gekennzeichnet. Das Opfer kann zu der Überzeugung kommen, dass etwas mit ihr nicht stimmt und dass sie dafür verantwortlich ist, etwas in sich selbst zu verändern, was Gewalt verhindern würde. Kognitive Reaktionen werden ausgelöst wie

RC: 101132

Verfügbar in:

https://www.nucleodoconhecimento.com.br/psychologie/bindungstrauma 
Selbstbeschuldigung, Schuld, Introjektion, Übertragung der Verantwortung für Missbrauch auf sich selbst und nicht auf den Aggressor, Konfiguration einer Darstellung von Ereignissen und sogar eine verzerrte Selbstdarstellung. Diese verzerrte Vorstellung kann vorübergehend als soziale Rechtfertigung für den Umgang mit Missbrauch und als rationale Erklärung dafür dienen, aber indem sie das Opfer dazu bringt, in der Beziehung fortzufahren, trägt eine solche Haltung dazu bei, dass das Opfer nicht in der Lage ist, den Kreislauf der Gewalt endgültig zu beenden (DUTTON; PAINTER, 1981).

Zwei Merkmale gewalttätiger Beziehungen tragen zur Bildung eines Bindungstrauma in der intimen Beziehung bei: das Ungleichgewicht der Macht und die eventuelle Intermitienz von Gewalt oder mit anderen Worten, der Zyklus der Gewalt, der mit der Phase der "Flitterwochen" durchsetzt ist. Das Ungleichgewicht der Macht tritt auf, wenn ein Individuum, das eine untergeordnete Rolle spielt, ein geringes Selbstwertgefühl entwickelt, seine Selbstwirksamkeit verringert und abhängiger vom gewalttätigen Partner, also der Person in einer dominanten Position (DUTTON; PAINTER, 1993).

Dazu kann auch eine patriarchale Gesellschaftsstruktur beitragen, die durch stereotype Geschlechterrollen gekennzeichnet ist. Wenn der Partner in der dominanten Position missbräuchlich ist, wird das Opfer emotional und existenziell eingesperrt. Dies erzeugt ein Gefühl der Hilflosen im Subjekt und stärkt die Bindung, weil das Opfer, das sich selbst als schwach sieht, die negative Wahrnehmung des Aggressors über inn verinnerlicht und inn noch abhängiger und machtloser macht, was eine starke affektive Bindung zu der Person schafft, die als stärker angesehen wird (SIMONIČ; OSEWSKA, 2019).

Die Abhängigkeit der person, die als zerbrechlicher wahrgenommen wird, verbirgt in der Tat die Abhängigkeit und Impotenz des Aggressors, weil sie auch vom Opfer abhängt. Aus diesem Grund unterbricht der Angreifer vorübergehend gewalttätiges Verhalten, aus Angst, die Grenze überschritten zu haben und das Opfer ihn verlässt.

RC: 101132

Verfügbar in:

https://www.nucleodoconhecimento.com.br/psychologie/bindungstrauma 
Die Angst, die beim Aggressor entsteht, führt zu einem Verhalten der Beschwichtigung, mit der Unterbrechung körperlicher und verbaler Gewalt, aber in der Regel immer noch unter Beibehaltung der psychologischen Manipulation. Auf diese Weise versucht der Aggressor, das Opfer an seiner Seite zu halten, wenn er mit der Möglichkeit konfrontiert wird, verlassen zu werden. Unter Verwendung der Theorie der Bindung, die intime Beziehungen zwischen Erwachsenen als Bindungsbeziehungen untersucht, ist die Angst, die mit der Möglichkeit einhergeht, die Person zu verlieren, an die sie gebunden ist, die Grundlage einer solchen pathologischen Beziehung (DUTTON; WHITE, 2012).

Laut Johnson (2008) sind Bindungsbedürfnisse in einer Beziehung gesund, aber im Falle von missbräuchlichen Beziehungen entsteht das Problem, wenn solche Bedürfnisse in einem Kontext der Unsicherheit geschaffen werden, der durch Konflikte erzeugt wird. Beziehungssicherheit hilft Menschen, ihre Emotionen zu regulieren, Informationen zu verarbeiten und klar zu kommunizieren. Diejenigen, die gesunde Bindungen haben, können ihre Unsicherheit offen zugeben und sich an andere wenden, um Unterstützung zu erhalten, Empfang und eine angemessene Reaktion auf die Situation zu erhalten.

Gewalt ist jedoch eine dysfunktionale Art, in der Nähe der Person zu bleiben, an der sie sich festhält, wenn das Bedürfnis nach Bindung geweckt wird. Das Opfer wird ängstlich, was es ihr schwer macht, die Beziehung zu verlassen, sowie ihr Aggressor, der die Kontrolle über das Opfer behalten möchte, damit sie ihn nicht verlässt (FINKEL; SLOTTER, 2007).

In diesem Fall tritt ein Paradoxon auf: Gewalt erhöht die Angst des Opfers, das fliehen möchte, während es das Bedürfnis nach Bindungen erhöht, die solche Qualen regulieren könnten, die sie bei Menschen sucht, die sie für stärker hält als sie. So wird der Aggressor zu einer Quelle der Angst und gleichzeitig des Schutzes. Die traumatische Bindung ist daher eine Quelle des Traumas sowie eine Bindung, die Sicherheit gibt (SIMONIČ; OSEWSKA, 2019).

RC: 101132

Verfügbar in:

https://www.nucleodoconhecimento.com.br/psychologie/bindungstrauma 
Ähnliche Dynamiken gibt es beim Stockholm-Syndrom, bei dem das Opfer eine starke emotionale Bindung zum Aggressor entwickelt. Dieses Syndrom, das auf kognitiven und Wahrnehmungsverzerrungen und Bindungen basiert, ist in diesem Zusammenhang ein Abwehrmechanismus, um mit solchen Faktoren umzugehen.

Bindungstrauma wird auch aufgrund einer bestimmten Aktivität auf organischer Ebene beider Partner in einer missbräuchlichen Beziehung gebildet und aufrechterhalten, die durch eine Dysregulation bei der Sekretion von Dopamin, endogenen Opioiden, Corticotrophin und Oxytocin gekennzeichnet ist, Hormone, die zur "Traumaabhängigkeit" beitragen. (BURKETT; YOUNG, 2012).

Oxytocin provoziert eine sehr starke neurologische Reaktion, die die Schaffung von Bindungen und den Aufbau von Vertrauen mit dem Partner fördert. Die Bindung und die Verbindung zwischen dem Opfer und dem Aggressor sind die gleichen wie die aller anderen Beziehungen: verstärkt durch überschüssiges Oxytocin.

Dopamin stimuliert das Verlangen, die Suche und das Samen für den anderen.

Endogene Opioide sind mit der Regulierung des Anteils von Vergnügen und Schmerz, Abstinenz und Abhängigkeit verbunden.

Die Freisetzung von Corticotrophin ist mit der Wahrnehmung von Stresssituationen und Stressreaktionen verbunden. Bei Gewalt werden in der Regel intensive neurochemische Prozesse aktiviert. Das Problem ist, dass solche neurobiologischen Prozesse, die die Bindung zum Stressabbau aktivieren, in toxischen und schädlichen Beziehungen auftreten, die auch eine Stressquelle sind, die diese Prozesse dereguliert und dazu führt, dass das Opfer zunehmend vom Aggressor abhängig wird, was einen Teufelskreis verursacht, wie den Zyklus häuslicher Gewalt (FISHER et al., 2010).

RC: 101132

Verfügbar in:

https://www.nucleodoconhecimento.com.br/psychologie/bindungstrauma 


\section{ENDGÜLTIGE ÜBERLEGUNGEN}

In Anbetracht des Vorstehenden, der zentralen Frage, die zu Beginn dieser Forschung über die Definition von Bindungstrauma und seine Mechanismen gemacht wurde, in einer Weise, dass man daraus schließen kann, dass mehrere emotionale und neuropsychobiologische Faktoren als Ursache für das Bindungstrauma innerhalb einer Beziehung dargestellt werden, und das Verständnis dieser Variablen grundlegend wird, sowohl für die therapeutische Ausarbeitung der traumatischen Bindung, als auch für den Prozess der Behandlung von Opfern und die Veränderung ihrer entsonanierenden und schädlichen Muster.

Darüber hinaus ist bekannt, dass eine erhöhte Anfälligkeit für die Bildung von Bindungstrauma in einer liebevollen Beziehung mit Personen mit einer Geschichte von Missbrauch und Gewalt in der Kindheit verbunden ist.

Solche frühen Lebenserfahrungen unterbrechen den natürlichen Prozess der Schaffung gesunder Bindungen und verändern die Gehirnstruktur des Subjekts, was zu einer größeren Anfälligkeit für eine missbräuchliche Beziehung führt, sowohl durch interne und hormonelle Faktoren als auch durch externe Faktoren, die ihm ein geringeres Gerät geben, um mit der Wahrnehmung von Gefahr und der Bildung positiver Bindungen umzugehen. sie fühlst dich untauglich, die Beziehung zu verlassen.

Zwischenmenschliche Regime bei erwachsenen Personen mit einer Geschichte von Missbrauch neigen dazu, negativ und in verschiedenen Beziehungen nicht testbar zu sein. Diese Schemata können Verhalten motivieren, das die Wahrscheinlichkeit einer erneuten Vvictimisierung erhöht.

So versteht man, dass das vorgeschlagene allgemeine Ziel: Bindungstrauma in liebevollen Beziehungen zu analysieren, um die neuropsychobiologischen Traumaprozesse, die in intimen Beziehungen erzeugt werden, zu gebären und die

RC: 101132

Verfügbar in:

https://www.nucleodoconhecimento.com.br/psychologie/bindungstrauma 
Gründe zu untersuchen, warum Opfer in pathologischen Beziehungen bleiben, klar in Betracht gezogen wurde.

Darüber hinaus wird angesichts all der theoretischen Verbindung, die gemacht wurde, angenommen, dass diese Studie eine signifikante Bedeutung hat, um einige Aspekte im Zusammenhang mit Bindungstrauma anzuführen, ohne das Thema jedoch zu erschöpfen. Daher soll dieser Artikel die Grundlage für weitere Studien sein, die an diesem Institut entwickelt werden sollen, die positiv zur Verbreitung von Wissen beitragen.

\section{VERWEISE}

AMERICAN PSYCHIATRIC ASSOCIATION - APA. Manual diagnóstico e estatístico de transtornos mentais: DSM-V. Porto Alegre: Artmed, 2014.

BLOOM, Sandra L. Trauma theory abbreviated. [s.I.: s.n.], 1999. Disponível em: https://strengthcounselling.ca/wp-content/uploads/2018/05/trauma-theoryabbreviated.pdf. Acesso em: 15 abr. 2021.

BURKETT, James P.; YOUNG, Larry J. The behavioral, anatomical and pharmacological parallels between social attachment, love and addiction. Psychopharmacology, v. 224, n. 1, p. 1-26, 2012. Disponível em: https://www.ncbi.nlm.nih.gov/pmc/articles/PMC3469771/. Acesso em: 15 dez. 2020.

CARNES, Patrick J. Betrayal Bond: Breaking Free of Exploitive Relationships. [s.l.]: Health Communications, Incorporated, 2019.

DEYOUNG, M.; LOWRY, JA. Traumatic bonding: clinical implications in incest. Child Welfare, 1992 Mar-Apr;71(2):165-175.

RC: 101132

Verfügbar in:

https://www.nucleodoconhecimento.com.br/psychologie/bindungstrauma 
DUTTON, Donald; PAINTER, S. L.Traumatic bonding: The development of emotional attachments in battered women and other relationships of intermittent abuse. Victimology, 6, 139-155, 1981.

DUTTON, Donald G.; PAINTER, Susan. Emotional Attachments in Abusive Relationships: A Test of Traumatic Bonding Theory. Violence and Victims. v. 8, n. 2, p. 105-120, 1993.

DUTTON, Donald G.; WHITE, Katherine R. Attachment insecurity and intimate partner violence. Aggression and Violent Behavior, v. 17, n. 5, p. 475-481, 2012.

FESTINGER, Leon. Cognitive Dissonance. Scientific American, v. 207, n. 4, p. 93106, 1962.

FISHER, Helen E.; et al. Reward, Addiction, and Emotion Regulation Systems Associated With Rejection in Love. Journal of Neurophysiology, v. 104, n. 1, p. 5160, 2010.

GEORGE, Vera. Traumatic Bonding and Intimate Partner Violence. [s.l.: s.n.], 2015. Disponível em: https://core.ac.uk/download/pdf/41339659.pdf. Acesso em: 23 Nov. 2020.

GRAHAM, D. L.; RAWLINGS, E. I.; IHMS, K.; LATIMER, D.; FOLIANO, J.; THOMPSON, A.; SUTTMAN, K.; FARRINGTON, M.; HACKER, R. A scale for identifying "Stockholm syndrome" reactions in young dating women: factor structure, reliability, and validity. Violence Vict, 1995 Spring;10(1):3-22. PMID: 8555117.

JOHNSON, M. P. A typology of domestic violence: intimate terrorism, violent resistance, and situational couple violence. Boston: Northeastern University Press, 2008.

RC: 101132

Verfügbar in:

https://www.nucleodoconhecimento.com.br/psychologie/bindungstrauma 
MARTíN, A. G. As sequelas psicológicas da tortura. Psicologia: Ciência e Profissão [online]. 2005, v. 25, n.3, pp. 434-449. Disponível em: <https://doi.org/10.1590/S1414-98932005000300008>. Acesso em: 22 Ago 2021.

SIMONIČ, Barbara; OSEWSKA, Elzbieta. Traumatic bonding in intimate partner violence: A Relational Family Therapy approach. Family Forum., v. 9, n. 10, 2019. 25167/FF/1092, p. 71-90, 2020.

VAN DER KOLK, Bessel A. The Compulsion to Repeat the Trauma. Psychiatric Clinics of North America, v. 12, n. 2, p. 389-411, 1989.

VAN DER KOLK, B. A. The Body Keeps the Score: Memory and the Evolving Psychobiology of Posttraumatic Stress. Harvard Review of Psychiatry, v. 1, n. 5, p. 253-265, jan. 1994.

VAN DER KOLK, Bessel A.; SAPORTA, Jose. The biological response to psychic trauma: Mechanisms and treatment of intrusion and numbing. Anxiety Research, v. 4, n. 3, p. 199-212, 1991.

VAN DER KOLK, B. A. The psychobiology and psychopharmacology of PTSD. Human Psychopharmacology: Clinical and Experimental, v. 16, n. S1, p. S49-S64, jan. 2001.

\section{ANHANG - REFERENZFUßNOTE}

3. Kostenlose Übersetzung der Autoren.

4. Kostenlose Übersetzung der Autoren.

5. Kostenlose Übersetzung der Autoren.

6. Kostenlose Übersetzung der Autoren.

RC: 101132

Verfügbar in:

https://www.nucleodoconhecimento.com.br/psychologie/bindungstrauma 
Eingereicht: Oktober 2021.

Genehmigt: November 2021.

RC: 101132

Verfügbar in:

https://www.nucleodoconhecimento.com.br/psychologie/bindungstrauma 